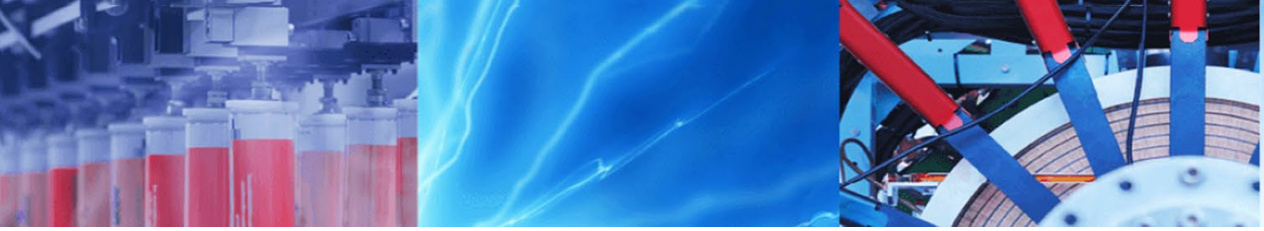

Research Article

\title{
Amorphous N-rich organic polymer/carbon nanotube composites as effective anode material for advanced lithium ion batteries
}

\author{
Shi-Xian $\mathrm{Xu}^{1} \cdot$ Wei $\mathrm{Xu}^{1} \cdot$ Ling-Jun Kong $^{1} \cdot$ Ying-Hui Zhang $^{1} \mathbb{D}$
}

Received: 9 September 2019 / Accepted: 6 January 2020 / Published online: 11 January 2020

(c) Springer Nature Switzerland AG 2020

\begin{abstract}
Amorphous N-rich porous organic polymer (SNW-1) was composited, respectively, with carboxylic carbon nanotubes (CNTs) and graphene (GE) through in situ synthesis, to construct new composites possessing both covalent porous network and enhanced conductivity. When used as anode of lithium ion batteries, the obtained chemically bonded SNW-1/ CNTs composite exhibits a capacity of $203 \mathrm{~mA} \mathrm{~h} \mathrm{~g}^{-1}$ at a current of $0.5 \mathrm{C}$ after 1000 cycles, which is much higher than that of SNW-1/GE $\left(72 \mathrm{~mA} \mathrm{~h} \mathrm{~g}^{-1}\right)$ and pristine SNW-1 $\left(73 \mathrm{~mA} \mathrm{~h} \mathrm{~g}^{-1}\right)$. This is interpreted in terms of different interaction between two components of composites. The chemical bonding of SNW-1/CNTs facilitates the heterogeneous charge transfer much more than the non-chemical interaction of SNW-1/GE, and thus gives a better electrochemical performance than the latter. This work provides a promising strategy to design organic polymer/carbonaceous materials composite for high-performance electrode.
\end{abstract}

Keywords SNW-1/CNTs composite $\cdot$ Lithium ion battery $\cdot$ Anode $\cdot$ Chemical bonding

\section{Introduction}

Lithium ion batteries (LIBs) have been widely used in modern digital devices [1-3]. Aiming to improve the electrochemical performance of LIBs, many efforts have been devoted to develop new kinds of functional materials for electrodes. There have many kinds of anode materials been reported till now, including carbonaceous materials, metal, phosphorus, metal oxides and metal sulfides [4-6]. Carbonaceous materials, for example graphene (GE) and carbon nanotubes (CNTs), are widely used as anode materials because of their high power density, good cycle performance and low cost [7-11]. However, there are still some defects for carbonaceous-based electrode, for example the irreversible capacity loss in the first cycle and the poor compatibility with electrolyte $[12,13]$. Furthermore, carbonaceous-based electrodes are apt to release combustible gas when operated at high temperature, which might lead to combustion or explosion of the electrochemical system [14]. To overcome these obstacles and furthermore improve the electrochemical performance of carbonaceous materials, many strategies have been developed, including heteroatoms $(\mathrm{N}, \mathrm{O}, \mathrm{S}$, and $\mathrm{P})$ doping [15-20] and composition with other functional materials [21-26].

Recently, porous organic polymers (POPs) have been reported as promising candidates in many applications, including adsorbent [27-29], drug release [30], photocatalyst [31-33], and lithium ion battery electrodes [34-39], owing to their large surface area and porous structure. Porous structure can effectively relieve the volume change of electrode during electrochemical processes,

Electronic supplementary material The online version of this article (https://doi.org/10.1007/s42452-020-1979-x) contains supplementary material, which is available to authorized users.

Ying-Hui Zhang, zhangyhi@nankai.edu.cn | 'School of Materials Science and Engineering, National Institute for Advanced Materials, Nankai University, Tianjin 300350, People's Republic of China. 
and thereby enhance the cycling stability. In addition, porous structure would reduce the diffusion length of ions and electrolyte, and hence improves the transport kinetics. Most of all, the high designability of POPs associated with versatile synthons facilitates the introduction of heteroatoms, which would provide much more accessible active sites for interacting with $\mathrm{Li}$ ion. However, the utilization of POPs as electrochemical materials is limited by their poor conductivity caused by lower conjugation degree. The compositization with carbonaceous materials is a facial method to overcome this obstacle, and the electrochemical performance of the resultant composite should associate closely with the interaction nature between POPs and carbonaceous material. To clarify this relationship, further investigations are required on newly fabricated composites.

As a cheap chemical, melamine is widely used in producing POPs. Feng and co-workers reported an amorphous N-rich POPs (SNW-1) synthesized through the polymerization of melamine and terephthalaldehyde, exhibiting large surface area and good chemical stability against most of common organic solvents [40]. Herein, we choose SNW-1 as porous material to composite with GE and carboxylic CNTs, to produce new electrochemically active composites (Scheme 1). When used as anode of LIBs. The obtained SNW-1/CNTs shows a specific capacity of $203 \mathrm{~mA} \mathrm{~h} \mathrm{~g}^{-1}$ after 1000 cycles at a current of $0.5 C$, which greatly surpasses that of SNW-1/GE and pristine SNW-1. The chemical interaction between two components in SNW-1/CNTs facilitates the heterogeneous charge transfer and accounts for the improved electrochemical performance.

\section{Experimental section}

\subsection{Materials and syntheses of SNW-1}

Carboxylic CNT was purchased from XFnano company. All other reagents are of analytical grade and used directly without further purification. SNW-1 was synthesized according to literature [40].

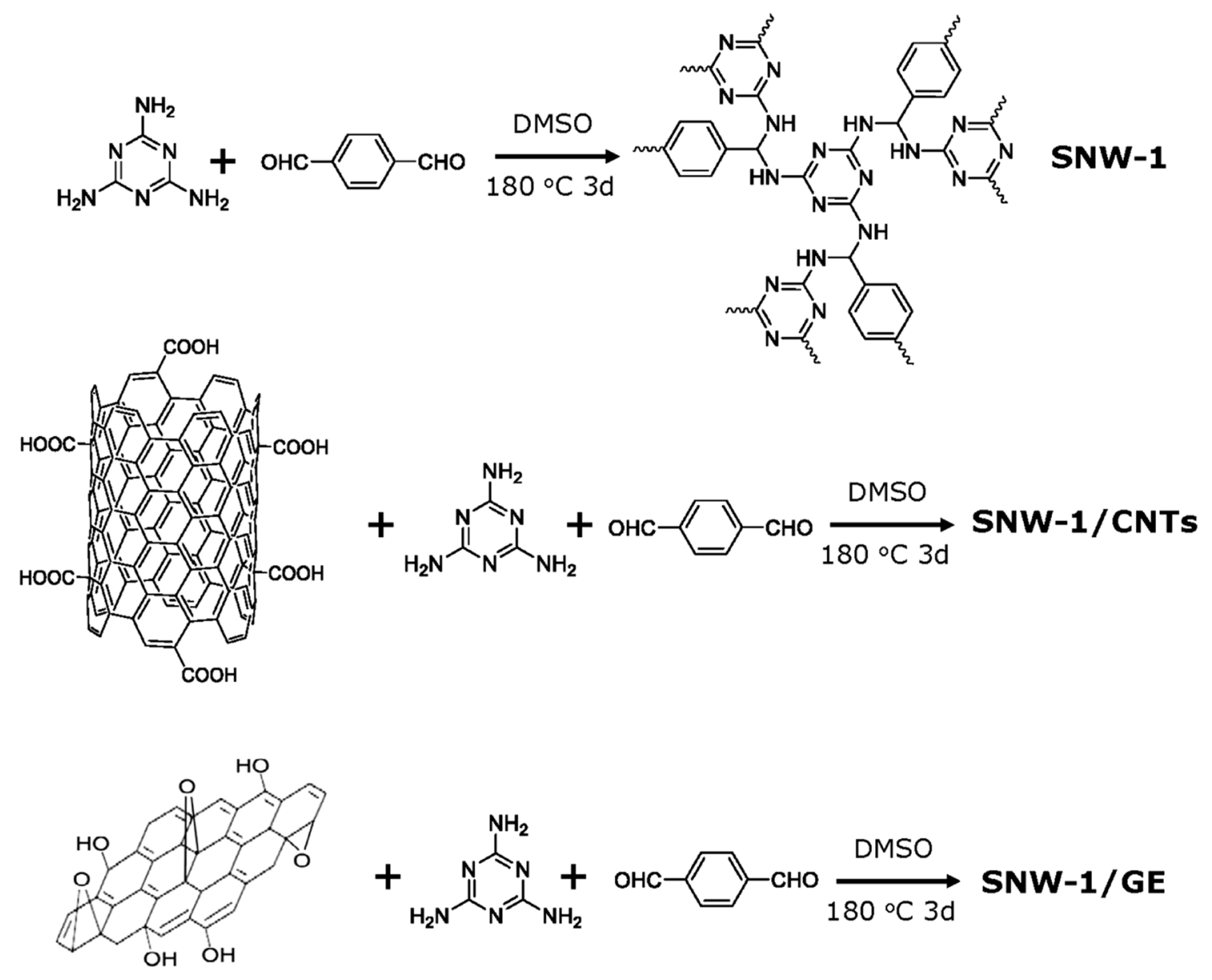

Scheme 1 Synthesis scheme of SNW-1, SNW-1/CNTs and SNW-1/GE 


\subsection{Syntheses of SNW-1/CNT}

A flame dried Schlenk flask fitted with a condenser and a magnetic stirring bar was charged with carboxylic CNT (10 mg), melamine (313 mg, $2.485 \mathrm{mmol})$, terephthalaldehyde (500 mg, $3.728 \mathrm{mmol}$ ) and dimethyl sulfoxide $(15.5 \mathrm{~mL})$. After being bubbled with argon for $10 \mathrm{~min}$, the mixture was heated at $180^{\circ} \mathrm{C}$ for $72 \mathrm{~h}$. Then the reaction system was cooled to room temperature, and the precipitated was collected by filtration and washed sequentially with acetone, tetrahydrofuran and dichloromethane. After being dried under vacuum at room temperature, black powder was obtained with a yield of $61 \%$.

\subsection{Syntheses of SNW-1/GE}

SNW-1/GE was produced in a similar procedure to that of SNW-1/CNT, excepting CNT being replaced by GE. The target SNW-1/GE composite was obtained as black powders in $64 \%$ yield.

\subsection{Structural characterization}

Fourier transform infrared (FTIR) spectra were recorded on a TENSOR 37 Frontier infrared spectrometer (Bruker, German) in $\mathrm{KBr}$ pellets. Thermogravimetric analysis (TGA) was carried out on a Rigaku standard TG-DTA analyzer with using empty $\mathrm{Al}_{2} \mathrm{O}_{3}$ crucible as reference, and the data were recorded between room temperature and $800{ }^{\circ} \mathrm{C}$ with a heating rate of $10^{\circ} \mathrm{C} \mathrm{min}^{-1}$ under air atmosphere. The morphology was characterized by SEM (JEOL JSM-7500F). XPS was measured on a PHI5000 Versaprobe system. Raman spectra were measured on Renishaw system in the range of $100-4000 \mathrm{~cm}^{-1}$. Gas sorption isotherms were measured on Micromeritics ASAP 2020 gas adsorption analyzer, and all samples were activated under a dynamic vacuum at $80^{\circ} \mathrm{C}$ for $8 \mathrm{~h}$ before measurement.

\subsection{Battery fabrication and electrochemical measurements}

The 2025 coin-type cells for electrochemical tests were assembled in an argon-filled glove box. The working electrodes were prepared by pasting the viscous slurry containing $60 \%$ active material, 20\% Ketjen Black, and 20\% poly(vinylidene fluoride) (PVDF) onto a copper foil current collector. The electrodes were dried in a vacuum oven at $110^{\circ} \mathrm{C}$ overnight to remove NMP solvent. Pure lithium foil was used as the counter electrode. $1 \mathrm{M} \mathrm{LiPF}_{6}$ dissolved in ethylene carbonate/dimethyl carbonate/diethyl carbonate (EC:DEC:DMC = 1:1:1, V\%) was used as electrolyte. The galvanostatic charge-discharge tests were performed on LAND battery testing system in a voltage range of
0.01-3.0 V (vs. Li/Li $\left.{ }^{+}\right)$. The EIS measurements were carried out on a Princeton VersaSTAT4 electrochemical workstation from $1 \mathrm{~Hz}$ to $100 \mathrm{kHz}$.

\section{Results and discussion}

\subsection{Structural characterization}

The composites were first characterized by FTIR spectra. As shown in Fig. 1a and Figure S1, the spectrum of SNW-1/GE composite is similar to that of pristine SNW-1, where the stretching vibration of triazine ring is clearly observed at 1550 and $1480 \mathrm{~cm}^{-1}$ [40], and the band at $3420 \mathrm{~cm}^{-1}$ may be assigned to the stretching vibration of $\mathrm{N}-\mathrm{H}$ bond of SNW-1. No bands attributable to the stretching vibration of imine bond (around $1600 \mathrm{~cm}^{-1}$ ) are observed. For SNW-1/ CNT system, the spectrum is dominated by the characteristic vibrations of CNTs component, while the IR bands of triazine ring are not obvious. Notably, the $1628 \mathrm{~cm}^{-1}$ band may be assigned to the amide bond formed through condensation between the carboxyl group of carboxylic CNTs and the amino groups of melamine. This chemical bonding would facilitate the charge transfer between CNTs and SNW-1, and further enhance the synergy effect.

The successful construction of SNW-1/CNT and SNW-1/ GE composites was also supported by SEM and Raman spectra measurement. The SEM of SNW-1/CNTs exhibits similar nanosheet morphology to that of SNW-1 (Figure S2), while the Raman spectrum shows the character $D$ $\left(1340 \mathrm{~cm}^{-1}\right)$ and $\mathrm{G}\left(1580 \mathrm{~cm}^{-1}\right)$ bands of CNTs (Figure S3). Similar results were also observed for SNW-1/GE (Figure S2 and S3). PXRD measurement does not unveil any diffraction peaks attributable to long range ordered structure, revealing the amorphous nature of two composites (Figure S4). In addition, TGA was used to evaluate the thermal stability of the materials. As shown in Fig. 1b, both SNW-1 and SNW-1/GE exhibit good thermal stability up to $350^{\circ} \mathrm{C}$ under air atmosphere, while SNW-1/CNT shows much high thermal stability up to $550^{\circ} \mathrm{C}$.

$\mathrm{X}$-ray photoelectron spectroscopy (XPS) measurements were performed to clarify the oxidation state of $\mathrm{N}, \mathrm{O}$, and $\mathrm{C}$ in the composites. As shown in Fig. 1 and Figure S5, similar $\mathrm{N} 1 \mathrm{~s}, \mathrm{C} 1 \mathrm{~s}$ and $\mathrm{O} 1 \mathrm{~s}$ spectra were observed for SNW-1/CNT, SNW-1/GE and SNW-1, which confirms similar assembly backbone of them.

Nitrogen gas adsorption/desorption isotherms recorded at $77 \mathrm{~K}$ were used to evaluate the porosity of SNW-1 and two composites (Fig. 2). All three samples exhibit type-I isotherm, indicating the microporous nature of them. The Brunauer-Emmett-Teller (BET) surface area was calculated to be 736,641 , and $723 \mathrm{~m}^{2} \mathrm{~g}^{-1}$, 

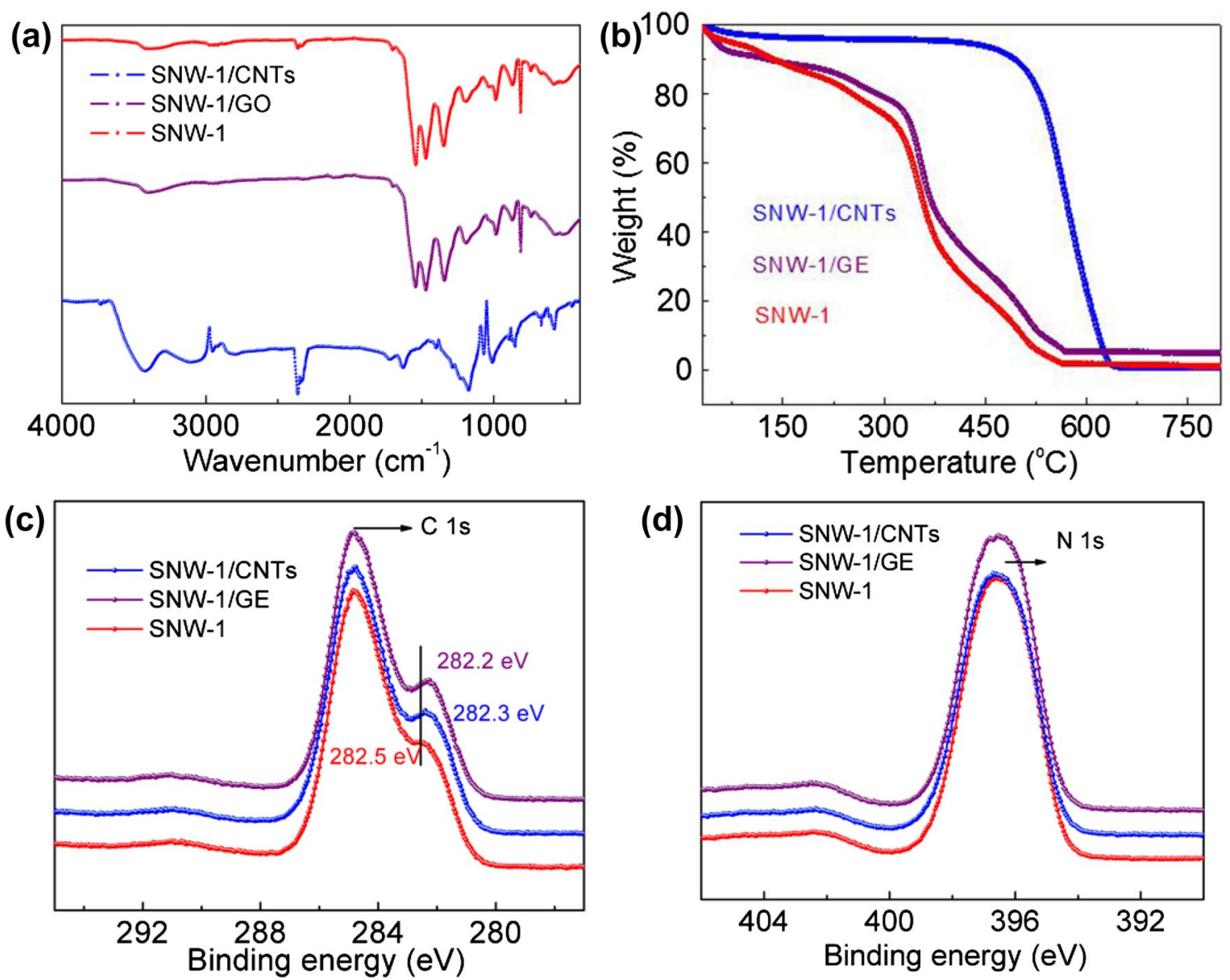

Fig. 1 a FTIR spectra of SNW-1, SNW-1/CNTs and SNW-1/GE; b TGA of SNW-1, SNW-1/CNTs and SNW-1/GE carried out between 30 and $800^{\circ} \mathrm{C}$ under air atmosphere; high-resolution XPS spectra of deconvoluted $\mathbf{c} \mathrm{N} 1 \mathrm{~s}$ and $\mathbf{d} \mathrm{C} 1 \mathrm{~s}$ peaks
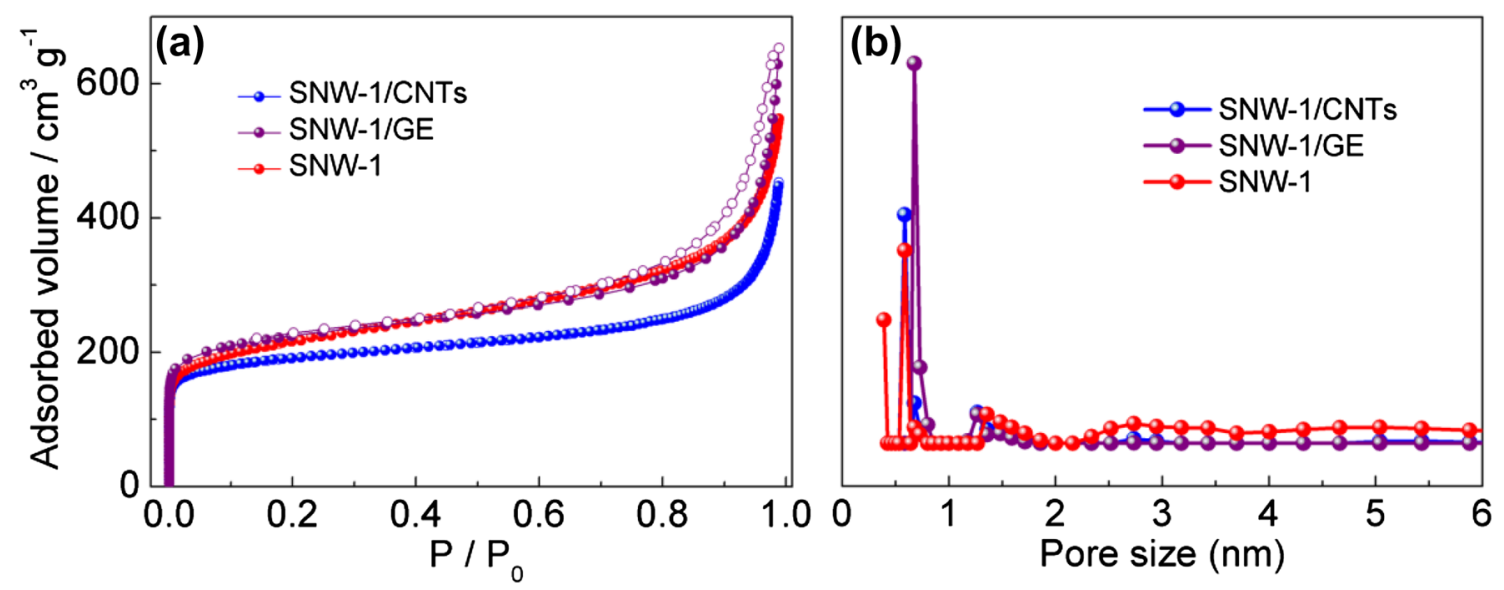

Fig. $2 \mathrm{~N}_{2}$ sorption isotherms of SNW-1, SNW-1/CNTs and SNW-1/GE (right panel: solid symbols, adsorption; open symbols, desorption) and the corresponding pore size distribution (left panel) calculated by non-local density functional theory (NLDFT) model

respectively, for SNW-1, SNW-1/CNTs and SNW-1/GE, and the main pore diameter was predicted to be less than $1 \mathrm{~nm}$ by non-local density functional theory (NLDFT) model. The large surface area should be attributed to the formation of porous SNW-1 component, which would provide more active sites readily accessible to $\mathrm{Li}^{+}$and electrolyte ions. 


\subsection{Performance of lithium ion batteries}

Owing to the high surface area, unique structure and composition advantages, the as-obtained samples could be ideal choice applied as anode materials in LIBs. The electrochemical impedance spectra (Nyquist plots) were firstly recorded to analyze the influence of compositization on the conductivity. As shown in Fig. 3, semicircle in the high-to-medium frequency region together with a straight line in the low frequency region was observed for all three samples. The former represents the charge-transfer resistance $\left(R_{c t}\right)$ that may be evaluated by the diameter of the semicircle, while the latter is associated with mass diffusion process [41]. Both SNW-1/CNTs and SNW-1/GE exhibit much smaller diameters than SNW-1, unveiling much improved conductivity of two composites relative to SNW-1 that makes it much easier for electron to transfer to the surface. This may be attributed to the high electrical conductivity of CNTs and GE components as well as the fine interfacial affinity between carbonaceous materials and SNW-1.

Given their large surface area, high nitrogen content and improved electrical conductivity, the as-synthesized composites were further investigated as anode candidates for LIBs. The cycling performances and Coulombic efficiencies of the two as-obtained SNW-1/CNTs and SNW-1/GE composites were tested and compared with that of SNW-1, as shown in Fig. 4a, c, e. When tested at a current density of $500 \mathrm{~mA} \mathrm{~g}^{-1}$, good cycling stabilities up to 1000 cycles are unveiled for all three materials. Notably, the SNW-1/ CNTs electrode delivers higher initial specific capacity of $134 \mathrm{mAh} \mathrm{g}^{-1}$ than SNW-1 $\left(90 \mathrm{mAh} \mathrm{g}^{-1}\right)$ and SNW-1/GE (55 $\mathrm{mAh} \mathrm{g}^{-1}$ ), respectively. After early activation process, the specific capacity of SNW-1/CNTs increases continuously

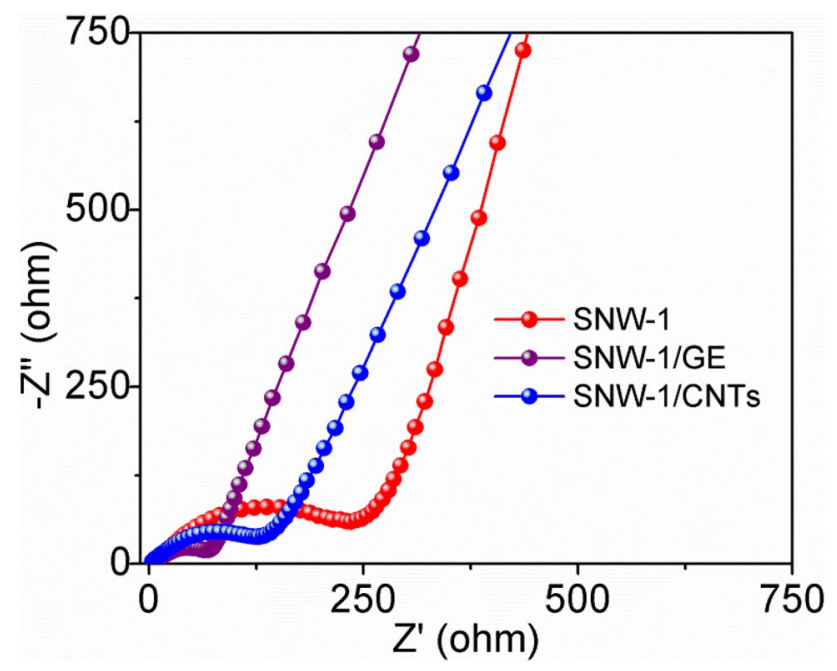

Fig. 3 The Nyquist plots of SNW-1, SNW-1/CNTs and SNW-1/GE and approaches to $203 \mathrm{mAh} \mathrm{g}^{-1}$ after 1000 cycles, which is about 1.5 times as high as the initial value. This could be attributed to the gradual release of electrochemical active sites existed in SNW component [42, 43]. The capacity increasing phenomenon was also observed for SNW-1 and SNW-1/GE but with much smaller steps, and the final specific capacities are about $75 \mathrm{mAh} \mathrm{g}^{-1}$ for both of them. The more effective activation process observed for SNW-1/ CNTs relative to SNW-1 and SNW-1/GE might be ascribed to the more strong interaction between two components in the former. Notably, the specific capacity of SNW-1 is comparable with that of some other organic polymers, including conjugated polymeric Schiff base (PSB) and conjugated poly(5-alkyl-thieno[3,4-c]pyrrole-4,6-dione)s $[44,45]$, while SNW-1/CNTs surpass the Gr/c-PMMA composites [46]. This could be attributed to the high content of $\mathrm{N}$ of SNW- 1 that favors the insertion of lithium ions. Furthermore, all three samples exhibit fascinating Columbic efficiency of up to $99 \%$ after 1000 cycles, further exhibiting excellent cycling performances.

In addition to the specific capacity and cycling stability, the rate performance of the as-formed SNW-1, SNW/ CNTs and SNW-1/GE electrodes were also explored and depicted in Fig. 4b, d, f, respectively, with current density varying from 0.025 to $10 \mathrm{~A} \mathrm{~g}^{-1}$. Along with current density increasing, the specific capacity of SNW-1 electrode fades severely from $50 \mathrm{mAh} \mathrm{h} \mathrm{g}^{-1}$ at $50 \mathrm{~mA} \mathrm{~g}^{-1}$ to $15 \mathrm{mAh}$ $\mathrm{g}^{-1}$ at $10 \mathrm{~A} \mathrm{~g}^{-1}$, while that of SNW-1/CNTs and SNW-1/ GE electrodes decrease from 132 and $126 \mathrm{mAh} \mathrm{g}^{-1}$ at 50 $\mathrm{mA} \mathrm{g}^{-1}$, respectively, both to both $48 \mathrm{mAh} \mathrm{g}^{-1}$ at $10 \mathrm{Ag}^{-1}$, indicating the better rate performance of two composites than the pristine SNW-1. In general, the rate performance associates closely with the conductivity, and the higher conductivity, the better rate performance [41]. In accordance with this tendency, the better rate performance of two composites should also be ascribed to their higher conductivity as observed in Fig. 3. When the current density is recovered to $0.1 \mathrm{~A} \mathrm{~g}^{-1}$, specific capacities of 55, 150, $125 \mathrm{mAh} \mathrm{g}^{-1}$ are obtained for SNW-1, SNW-1/CNTs, and SNW-1/GE electrodes, respectively, indicating the excellent cycling performances of these materials.

Besides rate performance, some other electrochemical performance such as charge/discharge specific capacity would also be improved by high conductivity. However, it's not the case for the three materials studied here. SNW-1/ GE exhibits unexpectedly the lowest capacity, though possessing the best electrical conductivity, whereas SNW-1/ CNT displays middle conductivity but the highest capacity. This abnormal case should be ascribed to different interaction between SNW-1 and carbonaceous components for the two composites. It has been indicated in IR analyses that SNW-1 interacts with carboxylic CNTs moiety through chemical bonding in SNW-1/CNTs composite, 

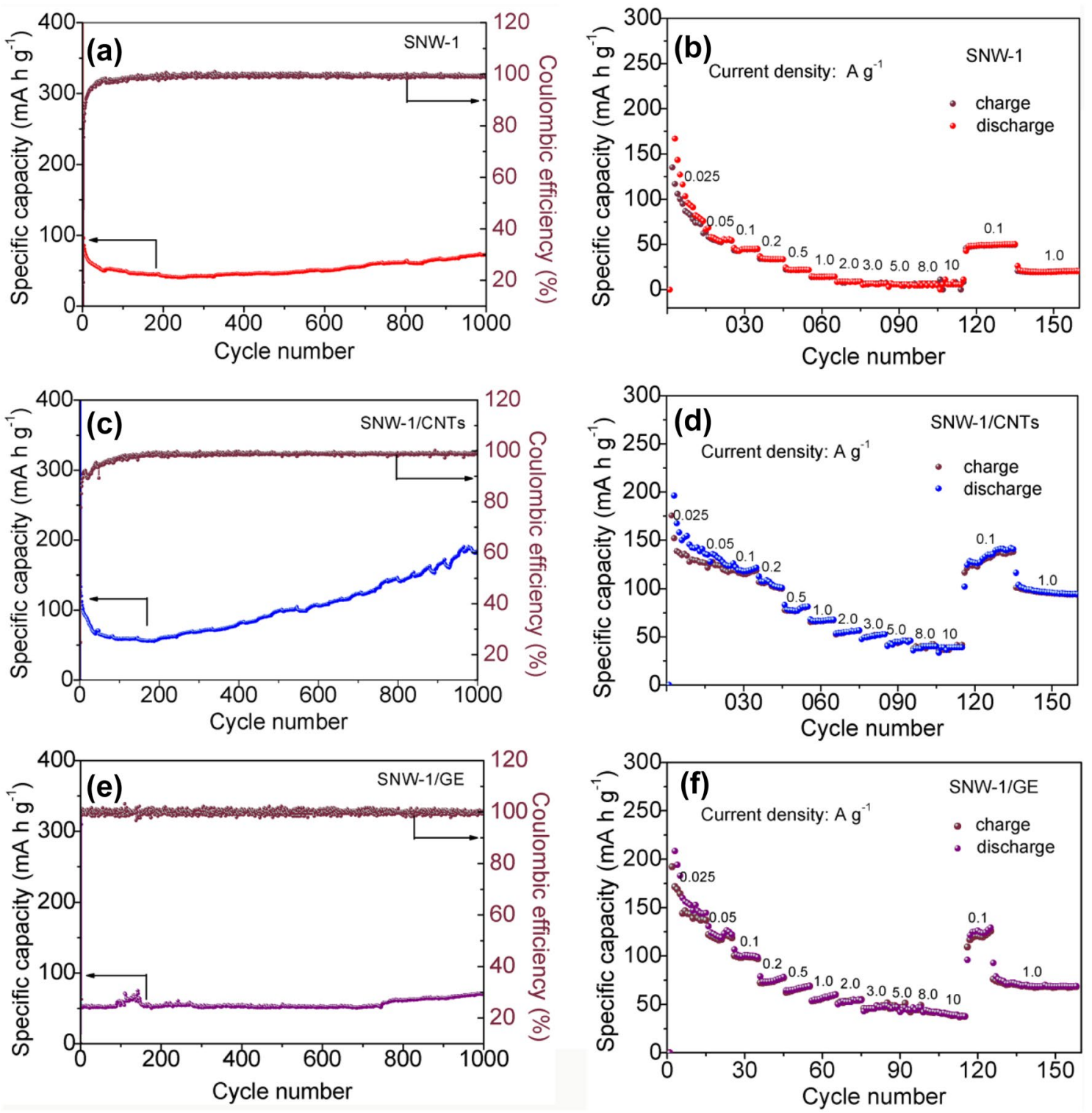

Fig. 4 Electrochemical behaviors of SNW-1, SNW/CNTs, and SNW-1/GE: a, c, e cycling performance and Coulombic efficiency at a current density of $500 \mathrm{~mA} \mathrm{~g}^{-1} ; \mathbf{b}, \mathbf{d}, \mathbf{f}$ rate performance at various current densities

which facilitates heterogeneous charge transfer during the repeating electrochemical processes, and thus would give high specific capacity. For SNW-1/GE, the non-bonding linkage between SNW-1 and GE component does not provide highly effective path for charge transfer, and thus the electrochemical reaction occurs separately over the surface of GE or over that of SWN-1. This depresses the synergy effect between two SNW-1 and GE components and consequently results in a poor electrochemical performance. To manifest this analysis, control experiment was carried out for a mechanical mixture of SNW-1 and
CNTs. As shown in Figure $\mathrm{S} 6$ and $\mathrm{S7}$, the mechanical mixture displays an initial capacity $\left(75 \mathrm{mAh} \mathrm{g}^{-1}\right)$ even smaller than that of pure SNW-1. After 1000 cycles, the capacity of the mixture approaches to $110 \mathrm{mAh} \mathrm{g}^{-1}$. Careful inspection reveals that the specific capacity of the mechanical mixture of SWN-1 and CNTs is only about half of that of SNW-1/CNTs composite, but is comparable to that of SWM-1/GE composite. This reveals that the interaction between different components of composites exerts an important influence on the corresponding electrochemical performance. 


\section{Conclusions}

In summary, a facile method was developed to construct new organic polymer/carbonaceous materials composites through in situ synthesis. When used as anode materials of LIBs, chemically bonded SNW-1/CNTs composite exhibits a good reversible capacity of $203 \mathrm{~mA} \mathrm{~h} \mathrm{~g}^{-1}$ after 1000 cycles at $0.5 \mathrm{~A} \mathrm{~g}^{-1}$, while SNW-1/GE composite assembled through nonchemical interaction shows a poor capacity, though possessing higher electronic conductivity. This indicates that electrochemical performance of the composite depends strongly on the heterogeneous interaction between different components. This work sheds new insight on constructing organic polymer-based composites for sustainable and environmentally energy storage devices.

Acknowledgements This work was supported by the NSFC (21421001 and 21531005)

\section{Compliance with ethical standards}

Conflicts of interest There are no conflicts to declare.

\section{References}

1. Armand M, Tarascon JM (2008) Building better batteries. Nature 451:652-657

2. Wang F, Wang J, Ren H, Tang H, Yu R, Wang D (2016) Multi-shelled LiMn $\mathrm{O}_{4}$ hollow microspheres as superior cathode materials for lithium-ion batteries. Inorg Chem Front 3:365-369

3. Poizot $P$, Dolhem $F$ (2011) Clean energy new deal for a sustainable world: from non- $\mathrm{CO}_{2}$ generating energy sources to greener electrochemical storage devices. Energy Environ Sci 4:2003-2019

4. Kim Y, Lee H, Kang S (2012) First-principles and experimental investigation of the morphology of layer-structured $\mathrm{LiNiO}_{2}$ and $\mathrm{LiCoO}_{2}$. J Mater Chem 22:12874-12881

5. Sun YK, Myung ST, Park BC, Prakash J, Belharouak I, Amine K (2009) High-energy cathode material for long-life and safe lithium batteries. Nat Mater 8:320-324

6. Hu LH, Wu FY, Lin CT, Khlobystov AN, Li LJ (2013) Graphenemodified $\mathrm{LiFePO}_{4}$ cathode for lithium ion battery beyond theoretical capacity. Nat Commun 4:1687

7. Wang Y, Kong D, Shi W, Liu B, Sim GJ, Ge Q, Yang HY (2016) Ice templated free-standing hierarchically $\mathrm{WS}_{2} / \mathrm{CNT}-\mathrm{rGO}$ aerogel for high-performance rechargeable lithium and sodium ion batteries. Adv Energy Mater 6:1601057

8. Xue DJ, Xin S, Yan Y, Jiang KC, Yin YX, Guo YG, Wan LJ (2012) Improving the electrode performance of Ge through Ge@C core-shell nanoparticles and graphene networks. J Am Chem Soc 134:2512-2515

9. Fang S, Shen L, Zheng H, Zhang X (2015) Ge-graphene-carbon nanotube composite anode for high performance lithium-ion batteries. J Mater Chem A 3:1498-1503

10. Zhong $M$, Zhang X, Yang D-H, Zhao B, Xie Z, Zhou Z, Bu X-H (2017) Zeolitic imidazole framework derived composites of nitrogen-doped porous carbon and reduced graphene oxide as high-efficiency cathode catalysts for $\mathrm{Li}-\mathrm{O}_{2}$ batteries. Inorg Chem Front 4:1533-1538

11. Kong L, Zhu J, Shuang W, Bu X-H (2018) Nitrogen-doped wrinkled carbon foils derived from MOF nanosheets for superior sodium storage. Adv Energy Mater 8:1801515

12. Cao W, Zheng J, Adams D, Doung T, Zheng JP (2014) Comparative study of the power and cycling performance for advanced lithium-ion capacitors with various carbon anodes. J Electrochem Soc 161:A2087-A2092

13. Nowak AP (2018) Composites of tin oxide and different carbonaceous materials as negative electrodes in lithium-ion batteries. J Solid State Electrochem 22:1-8

14. Zeng K, Gauthier D, Soria J, Mazza G, Flamant G (2017) Solar pyrolysis of carbonaceous feedstocks: a review. Sol Energy 156:73-92

15. Li J, Xue C, Xi B, Mao H, Qian Y, Xiong S (2018) Heteroatom dopings and hierarchical pores of graphene for synergistic improvement of lithium-sulfur battery performance. Inorg Chem Front 5:1053-1061

16. Xiao J, Mei D, Li X, Xu W, Wang D, Graff GL, Liu J (2011) Hierarchically porous graphene as a lithium-air battery electrode. Nano Lett 11:5071-5078

17. Li Y, Zhang H, Wang Y, Liu $P$, Yang $H$, Yao X, Zhao H (2014) A selfsponsored doping approach for controllable synthesis of $S$ and $\mathrm{N}$ co-doped trimodal-porous structured graphitic carbon electrocatalysts. Energy Environ Sci 7:3720-3726

18. Wasalathilake KC, Ayoko GA, Yan C (2018) Effects of heteroatom doping on the performance of graphene in sodium-ion batteries: a density functional theory investigation. Carbon 140:276-285

19. Xiao Z, Song Q, Guo R, Kong D, Zhou S, Huang X, Zhi L (2018) Nitrogen-enriched carbon/CNT composites based on Schiffbase networks: ultrahigh $\mathrm{N}$ content and enhanced lithium storage properties. Small 14:1703569

20. Liu H, Tang Y, Zhao W, Ding W, Xu J, Liang C, Huang F (2018) Facile synthesis of nitrogen and halogen dual-doped porous graphene as an advanced performance anode for lithium-ion batteries. Adv Mater Interfaces 5:1701261

21. Sasikala SP, Lim J, Kim IH, Jung HJ, Yun T, Han TH, Kim SO (2018) Graphene oxide liquid crystals: a frontier 2D soft material for graphene-based functional materials. Chem Soc Rev 47:6013-6045

22. Wang B, Guo R, Zheng M, Liu Z, Li F, Meng L, Jiang H (2018) Embedded binary functional materials/cellulose-based paper as freestanding anode for lithium ion batteries. Electrochim Acta 260:1-10

23. Wang G, Zhang J, Yang S, Wang F, Zhuang $X$, Müllen $K$, Feng $X$ (2018) Vertically aligned $\mathrm{MoS}_{2}$ nanosheets patterned on electrochemically exfoliated graphene for high-performance lithium and sodium storage. Adv Energy Mater 8:1702254

24. Haldar S, Roy K, Nandi S, Chakraborty D, Puthusseri D, Gawli Y, Vaidhyanathan R (2018) High and reversible lithium ion storage in self-exfoliated triazole-triformyl phloroglucinol-based covalent organic nanosheets. Adv Energy Mater 8:1702170

25. Xu F, Jin S, Zhong H, Wu D, Yang X, Chen X, Jiang D (2015) Electrochemically active, crystalline, mesoporous covalent organic frameworks on carbon nanotubes for synergistic lithium-ion battery energy storage. Sci Rep 5:8225

26. Yang DH, Yao ZQ, Wu D, Zhang YH, Zhou Z, Bu XH (2016) Structure-modulated crystalline covalent organic frameworks as high-rate cathodes for Li-ion batteries. J Mater Chem A 4:18621-18627

27. Song W-C, Xu X-K, Chen Q, Zhuang Z-Z, Bu X-H (2013) Nitrogenrich diaminotriazine-based porous organic polymers for small gas storage and selective uptake. Polym Chem 4:4690-4696 
28. Xiang Z, Mercado R, Huck J, Wang H, Guo Z, Wang W, Cao D, Haranczyk M, Smit B (2015) Systematic tuning and multi-functionalization of covalent organic polymers for enhanced carbon capture. J Am Chem Soc 137:13301-13307

29. Yang S-J, Ding X, Han B-H (2018) Conjugated microporous polymers with extended pi-structures for organic vapor adsorption. Macromolecules 51:947-953

30. Zhao H, Jin Z, Su H, Jing X, Sun F, Zhu G (2011) Targeted synthesis of a $2 \mathrm{D}$ ordered porous organic framework for drug release. Chem Commun 47:6389-6391

31. Wang L, Wan Y, Ding Y, Wu S, Zhang Y, Zhang X, Zhang G, Xiong Y, Wu X, Yang J, Xu H (2017) Conjugated microporous polymer nanosheets for overall water splitting using visible light. Adv Mater 29:1702428

32. Lin G, Ding H, Chen R, Peng Z, Wang B, Wang C (2017) 3D porphyrin-based covalent organic frameworks. J Am Chem Soc 139:8705-8709

33. Zhang G, Lin L, Li G, Zhang $Y$, Savateev A, Zafeiratos $S$, Wang $X$, Antonietti M (2018) lonothermal synthesis of triazine-heptazine based coframeworks with apparent quantum yields of $60 \%$ at $420 \mathrm{~nm}$ for solar hydrogen production from sea water. Angew Chem Int Ed 57:9372-9376

34. Roberts AD, Li X, Zhang H (2014) Porous carbon spheres and monoliths: morphology control, pore size tuning and their applications as Li-ion battery anode materials. Chem Soc Rev 43:4341-4356

35. Miroshnikov M, Divya KP, Babu G, Meiyazhagan A, Arava LMR, Ajayan PM, John G (2016) Power from nature: designing green battery materials from electroactive quinone derivatives and organic polymers. J Mater Chem A 4:12370-12386

36. Zhang C, He Y, Mu P, Wang X, He Q, Chen Y, Jiang JX (2018) Toward high performance thiophene-containing conjugated microporous polymer anodes for lithium-ion batteries through structure design. Adv Funct Mater 28:1705432

37. Alkarmo W, Aqil A, Ouhib F, Thomassin JM, Mazouzi D, Guyomard D, Jérôme C (2017) Nanostructured 3D porous hybrid network of $\mathrm{N}$-doped carbon, graphene and Si nanoparticles as an anode material for Li-ion batteries. New J Chem 41:10555-10560

38. Wang HG, Yuan C, Zhou R, Duan Q, Li (2017) Self-sacrifice template formation of nitrogen-doped porous carbon microtubes towards high performance anode materials in lithium ion batteries. Chem Eng J 316:1004-1010

39. Wang HG, Wu Z, Meng FL, Ma DL, Huang XL, Wang LM, Zhang XB (2013) Nitrogen-doped porous carbon nanosheets as lowcost, high-performance anode material for sodium-ion batteries. ChemSusChem 6:56-60

40. Schwab MG, Fassbender B, Spiess HW, Thomas A, Feng X, Mullen K (2009) Catalyst-free preparation of melamine-based microporous polymer networks through Schiff base chemistry. J Am Chem Soc 131:7216-7217

41. Xie X, Ao Z, Su D, Zhang J, Wang G (2015) MoS $_{2}$ /graphene composite anodes with enhanced performance for sodium-ion batteries: the role of the two-dimensional heterointerface. Adv Funct Mater 25:1393-1403

42. Zou F, Chen Y-M, Liu K, Yu Z, Liang W, Bhaway SM, Gao M, Zhu $Y(2016)$ Metal organic frameworks derived hierarchical hollow $\mathrm{NiO} / \mathrm{Ni} / g r a p h e n e$ composites for lithium and sodium storage. ACS Nano 10:377-386

43. Kang H, Liu H, Li C, Sun L, Zhang C, Gao H, Yin J, Yang B, You Y, Jiang K-C, Long $\mathrm{H}$, Xin S (2018) Polyanthraquinone-triazine: a promising anode material for high-energy lithium-ion batteries. ACS Appl Mater Interfaces 10:37023-37030

44. Ye H, Jiang F, Li H, Xu Z, Yin J, Zhu H (2017) Facile synthesis of conjugated polymeric Schiff base as negative electrodes for lithium ion batteries. Electrochim Acta 253:319-323

45. Robitaille A, Perea A, Bélanger D, Leclerc M (2017) Poly(5-alkylthieno[3,4-c]pyrrole -4,6-dione): a study of p-conjugated redox polymers as anode materials in lithium-ion batteries. J Mater Chem A 5:18088-18094

46. Sha Z, Qiu S, Zhang Q, Huang Z, Cui X, Yang Y, Lin Z (2019) A facile solvothermal polymerization approach to thermoplastic polymer-based nanocomposites as alternative anodes for high-performance lithium ion batteries. J Mater Chem A 7:23019-23027

Publisher's Note Springer Nature remains neutral with regard to jurisdictional claims in published maps and institutional affiliations. 